\title{
Dermatotenodesis technique in conjunction with a blood- patch treatment; A combined solution concerning tissue loss with exposed tendon in a case of ischaemic foot wound
}

\author{
Claudina Rudolph ${ }^{1 *}$, Kjeld Bull Skafdrup ${ }^{2}$, Birgit Dashnaw ${ }^{3}$ and Annette Hogh ${ }^{4}$ \\ ${ }^{1}$ Skejby Univeristy Hospital Aarhus, Department of vascular surgery, Region East Jutland, Denmark \\ ${ }^{2}$ Orthopaedic surgeon, Regional Hospital Viborg, Region Central Jutland, Denmark \\ ${ }^{3}$ Nurse specializing in wound therapy, Regional Hospital Viborg, Region Central Jutland, Denmark \\ ${ }^{4}$ Ass. Professor, Regional Hospital Viborg, Department of vascular surgery, Region Central Jutland, Denmark
}

\begin{abstract}
Complex cases of ischemic foot wounds can be extremely challenging and medically complicated. Additional treatments to standard care thereby often become critical as well as essential in achieving a more patient relevant and clinically satisfactory resolution. To optimize net patient benefit and satisfaction, an inter-professional healthcare teamwork concept, defined as a multidisciplinary team (MDT), is instrumental in our standard patient care. In terms of this case, it proved to be a groundbreaking factor with regard to the final clinical conclusion and the outcome from the patient's perspective, which is equally desirable.
\end{abstract}

The case is based on a patient with universal arteriosclerosis, who developed rest pain and an ischemic ulceration. In addition, the tibialis anterior tendon was exposed as well as the cuneiform bone on the dorsum of the foot.

Despite the presence of sufficient revascularization and the application of standard wound care, an MDT approach proved vital in achieving limb salvage, optimal rehabilitated limb function and a more patient acceptable outcome.

More specifically, in addition to standard wound care, an innovative combined solution was employed. A dermatotenodesis technique and an autologous blood-patch treatment were applied together. This was a fundamental factor in the battle of achieving healing and limb restoration to an acceptable functional level for the patient.

\section{Background}

Critical limb threatening ischemia (CLTI) is a condition caused by arterial insufficiency. It is defined by the presence of ischemic rest pain, non-healing ulcers or gangrene in the limbs [1]. CLTI is the end stage of peripheral arterial disease attributable to universal arteriosclerosis caused by risk factors, such as like hypercholesterolemia, smoking, hypertension and diabetes [1,2]. Revascularisation and standard wound care (including off-loading, infection- and oedema control) are the golden standard to achieve pain relief, limb salvage and functional acceptable outcome for the patient.

Complex clinical problems in patients with CLTI demand a comprehensive solution as a supplement to standard care. Implementation of a multidisciplinary wound team (MDT), engaging a specialised patient defined inter-professional healthcare teamwork concept, provided a more professionally well-rounded perspective. MDT members independently treat various issues a given patient may have by focussing on the issues with which they specialise [3]. When multiple competences unite. It then becomes possible to see the patient from several critical viewpoints, thus creating the opportunity for ground-breaking innovation.

In this case, the local MDT tackled the situation by applying an innovative combination composed of an autologous blood-patch treatment [4] and the application of a dermatotenodesis technique to the standard wound treatment. The patient suffered from a wound caused by CLTI with exposed tendons and bone.

The autologous blood-patch is a three-layered structure, consisting of leucocytes, fibrin and platelets. It is created from $18 \mathrm{~mL}$ of the patient's venous blood. The blood is centrifuged for $8 \mathrm{~min}$. It then coagulates for approximately $10 \mathrm{~min}$. prior to compaction for $2 \mathrm{~min}$. at which time a patch is produced. The entire process takes approximately $20 \mathrm{~min}$. in total. The patch is then placed directly on to the surface of the ulcer, which afterwards is covered by a low adherent protective dressing [4]. The bloodpatch acts by stimulating wound healing and infection control by releasing cytokines and growth-factors. Due to the increased concentration of blood cells an accelerated healing is potentially provided.

The dermatotenodesis technique is a known, but not used standard surgical procedure in the orthopaedic regime, which is used to treat fingertip injuries, especially concerning "mallet fingers". The

${ }^{\star}$ Correspondence to: Claudina Rudolph, Palle Juul Jensens Blvd. 99, 8200 Aarhus, Denmark, E-mail: claudina1802@hotmail.com

Key words: dermatotenodesis, blood-patch treatment, multidisciplinary team (MDT), critical limb threatening ischemia (CLTI), wound management

Received: April 17, 2021; Accepted: April 22, 2021; Published: April 26, 2021 
characteristics of a "mallet finger" is the disruption of the extensor mechanism resulting in the inability to actively extend the distal interphalangeal joint (DIPJ) [5]. The dermatotenodesis technique immobilises the area by fixating the proximal and distal tendon to the healthy skin/subcutaneous tissue in an easy passive extension of the DIPJ.

Kirschner wires, across the DIPJ, secure the area to be held in extension [5]. The dermatotenodesis technique was extrapolated to this case to immobilise the extensor mechanism of the foot combined with immobilisation of the ankle joint using an air cast, which provided "removable off-loading".

This case report emphases the importance of an MDT approach in complex cases of CLTI were alternative methods become necessary to avoid major amputation or loss of walking skills.

\section{Case report}

A 77-year-old male, non-diabetic smoker with a former history of coronary-artery bypass and endovascular surgery with kissing stents developed rest pain and an ischemic ulceration on the dorsum of his right foot (toe pressure equivalent to $23 \mathrm{mmHg}$ ). An inconclusive CT angiography delayed the diagnostic process, and the following MR angiography prolonged the diagnostic process by one week, thus causing tissue lost on the ischemic foot and accelerated ischemic pain. This induced compression failure and postponed the necessary vascular reconstruction due to oedema.

The MRI showed open central vessels, occlusion of the superficial femoral artery and periodically stenosis of the supragenicular popliteal artery. The infragenicular popliteal artery, posterior tibial artery (ATP) and peroneal artery were open. Revascularisation was performed with a bypass from the common femoral artery to ATP. This resulted in a palpable pulse in the bypass graft as well as in the ATP below the medial malleolus. A toe pressure equivalent to $57 \mathrm{mmHg}$ was measured on the patient's right toe after the bypass. Despite successful revascularization the necrosis expanded on the dorsum of the foot including the distal tibialis anterior tendon, the extensor halluces and the extensor digitorum.

All necrotic tissue, including the extensor tendons, were surgically removed followed by immobilisation of the ankle joint in an air cast. Regardless of optimal standard wound care along with repeated surgical revisions, no progress in wound healing was seen.

Furthermore, the patient's tibialis anterior tendon was exposed in combination with development of bone contact to the proximal part of the first metatarsal bone and the medial cuneiform bone. The patient was facing the threat of foot drop or major amputation.

To elucidate patient-specific treatment options the local MDT comprised of a vascular surgeon, an orthopaedist specializing in ankle/ foot surgery, wound care specialists and a podiatrist were gathered. The MDT's conferred the case with the collaborating department of plastic surgery but no functional reconstructive possibilities were evident. A combination of an autologous blood-patch treatment (to stimulate granulation; also, over the exposed bone and tendon) and a dermatotenodesis of the tibialis anterior tendon were initiated in addition to immobilisation in the air cast. The dermatotenodesis was performed with a non-absorbable consecutive suture without lock through both tendon and vital skin. The combination of advanced wound treatment, in correlation with immobilisation and a bloodpatch, stimulated the formation of granulation tissue over the exposed tendon. The skin in this area healed completely.

During the process a good cooperation between the primary sector and the MDT was established, which created more flexibility for the patient. During the time period after revascularisation the patient was seen once pr. week for the blood-patch treatments.

Thereafter, the frequency of hospital visits decreased. The weekly treatments were accomplished through telecommunication between the primary sector and the MDT.

In summary, a highly successful result was achieved after 10 treatments with a new blood-patch weekly and one replacement of the suture after the initial dermatotenodesis procedure to create more stability. Regarding the quality of functional outcome for the patient, he regained full use of his limb without prosthetic assistance and was provided custom-made insoles to prevent foot ulcer recurrence (Figure 1).

\section{Discussion}

When facing a patient case with a complex ischemic wound, the importance of collaboration and alliances across specialties is indisputable. This case report elucidates the importance of an MDT approach allowing multiple competences to meet so the essence of innovation can evolve.

Successful revascularization was not sufficient to avoid major amputation in this case. A combination of advanced wound healing strategies came into play due to the MDT approach.

The blood-patch method was tested in a blinded controlled study on a diabetes population with foot ulcers against standard wound care. Within 20 weeks $34 \%$ in the LeucoPatch group healed vs. $22 \%$ in the standard care group, giving an unadjusted odds ratio (OR) of $1.58(96 \%$ CI 1.04-2.40, $\mathrm{p}=0.0235$ ) for healing in the intention-to-treat population [4]. The response is due to the release of cytokines and growth factors, which stimulates angiogenesis, tissue repair and infection control as well as similar blood cells, so the concentration of blood cells available in the tissues increases. This composition of blood cells is made possible by performing a blood patch as the study above realizes.

Exposed tendons are a classic problem seen in both orthopaedic and vascular regimes because of compromised blood supply. As a consequence of CLTI our case had the extensor tendons removed contemporary with the exposed tibialis anterior tendon remaining. The removal was based on necrotic tissue and not lack of functionality. This combination could have serious consequences for the mobility of the patient, for example, with foot drop or major amputation as a potential risk. Loss of the tibialis anterior tendon will result in limited ability of lifting the foot, which compromises the main function of walking therefore technically foot drop is developed. Foot drop is defined as significant weakness of foot and ankle dorsiflexion due to motor function loss. The dorsiflexors include the tibialis anterior, the extensor digitorum longus and the extensor halluces longus [6].

\section{Conclusion}

In this case, an innovative solution combining a dermatotenodesis and autologous blood-patch treatment, proved ground-breaking in the battle to achieve healing and restoring of the functional gait level in this case. 


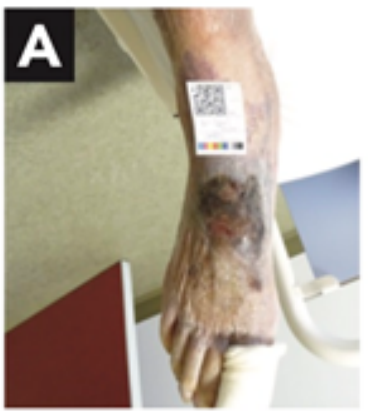

A) $27 / 1.19$

Ischemic wound with cyanotic skin before in situ bypass.

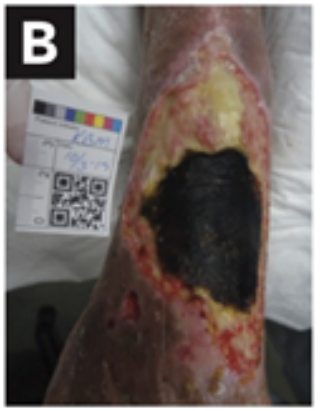

B) 10/3-19

Wound $12 \times 9 \mathrm{~cm}$ with the tendon proximal, In situ bypass performed 29.01.19.

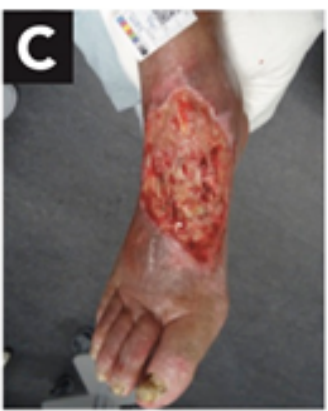

C) $26 / 3-19$

Debridement followed by exposed tendon.

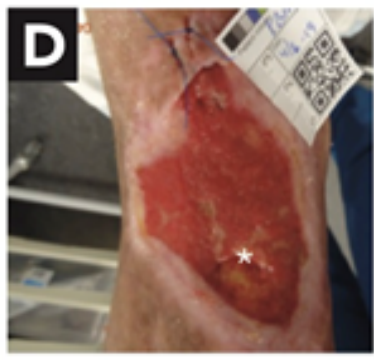

D) $4 / 6-19$

Wound $6 \times 9 \mathrm{~cm}$, exposed bone $(") 1 \times 1 \mathrm{~cm}$ The tendon is sutured proximal 21,05.19

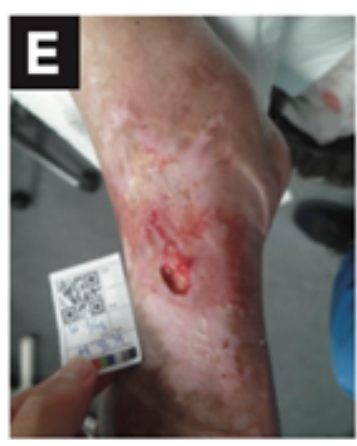

\section{E) $30 / 7-19$}

6 months postoperatively ind, 10 leucopatch treatments and derma. totenodese, wound $1.7 \times 1 \mathrm{~cm}$ and no exposed tendon.

Figure 1. Timeline of wound development

Complex cases with CLTI, especially with exposure of indispensable tendons, can be challenging and necessitate innovative treatments to standard care. This case elucidates the effective approach by the means of an MDT.

\section{Informed Consent} report.

Written informed consent was obtained from the patient in the case

\section{Declaration of conflicting interests}

The authors declared no potential conflicts of interest with respect to the research, authorship, and/or publication of this article.

\section{Funding}

The authors received no financial support for the research, authorship, and/or publication of this article.

\section{References}

1. Conte MS, Bradbury AW, Kolh P, White JV, Dick F, et al (2019) Global Vascular Guidelines on the mangement of chronic limb-threatening ischemia. $J$ Vasc Surg 69: 3S-125S. [Crossref]

2. Clair D, Shah S, Weber J (2012) Current State of Diagnosis and Management of Critical Limb Ischemia. Curr Cardiol Rep 14: 160-170. [Crossref]

3. Moore Z, Butcher G, Corbett LQ, McGuiness W, Snyder RJ, et al. (2014) Managing Wounds as a Team. J Wound Care 23: S1-S38.

4. Game F, Jeffcoate W, Tarnow L, Jacobsen JL, Whitham DJ, et al. (2018) LeucoPatch system for the management of hard-to-heal diabetic foot ulcers in the UK, Denmark, and Sweden: an observer-masked, randomised controlled trial. Lancet Diabetes Endocrinol 6: 870-878. [Crossref]

5. Kardestuncer T, Bae DS, Waters PM (2008) The results of tenodermodesis for severe chronic mallet [inger deformity in children. J Pediatri Orthop 28: 81-85. [Crossref]

6. Oktem NB, Tari R, Kotil K, Bilge T (2010) Cerebral Contusion as a Rare Cause of Foot Drop: Case Report. Turk Neurosurg 22: 99-101. [Crossref]

Copyright: (C2021 Rudolph C. This is an open-access article distributed under the terms of the Creative Commons Attribution License, which permits unrestricted use, distribution, and reproduction in any medium, provided the original author and source are credited. 